Case report

\title{
Neuropsychological diagnostics of neurosarcoidosis
}

\author{
Vera B. Nikishina ${ }^{1}$, Yuliya V. Zolotukhina ${ }^{2}$, Sergey A. Sirotkin ${ }^{2}$, Vitaliy B. Laskov ${ }^{1}$, Ekaterina A. Petrash ${ }^{1}$ \\ ${ }^{1}$ Kursk State Medical University, Kursk, Russia \\ ${ }^{2}$ Kursk Medical Sanitary Unit of the Ministry of Internal Affairs of the Russian Federation, Kursk, Russia
}

Received 24 October 2016, Revised 23 October 2017, Accepted 25 November 2017

(C) 2017, Nikishina V.B., Zolotukhina Y.V., Sirotkin S.A., Laskov V.B., Petrash E.A.

(C) 2017, Russian Open Medical Journal

Abstract: This article presents the results of neuropsychological studies of the patients with neurosarcoidosis. The obtained results show that typical manifestations of neurosarcoidosis are disorders of motor functions. They appear in the speed reduction of actions and their accuracy, which prove peripheral neuropathy and myopathy at the functional level as clinical signs of neurosarcoidosis. Specific manifestations of neurosarcoidosisare determinedby topographical localization of granulomatoma.

Keywords: neurosarcoidosis, neuropsychological assessment, clinical case

Cite as Nikishina VB, Zolotukhina YV, Sirotkin SA, Laskov VB, Petrash EA. Neuropsychological diagnostics of neurosarcoidosis. Russian Open Medical Journal 2018; 7: e0108.

Correspondence to Ekaterina A. Petrash. E-mail: petrash@mail.ru.

\section{Introduction}

Neurosarcoidosis is one of the manifestations of systemic sarcoidosis, which is a progressive nervous system impairment [1]. Occurrence of neurosarcoidosisis 8-10 cases per 100,000 people according to the data given by the modern researchers [2].

Incidence varies in different countries from 4 to 47 casesper 100,000 people [3-8]. The research surveys show a positive dynamics of neurosarcoidosis incidence [1]. Neurosarcoidosis distributes among people of all age groupsbut people at the age of 34-40 are mostly prone to the disease [3-5].

The clinical symptoms of the nervous system involvementare detected in $5-10 \%$ of patients with sarcoidosis [2]. As a rule, neurosarcoidosis develops among patients with confirmed clinical diagnosis of sarcoidosis. The neurological disorders can be the primary manifestation in less than half of the cases, and in $10 \%$ of the cases they areonly sign of the disease without any other organs involvement [2]. Nowadays the statistics on incidence of neurosarcoidosisis not carried out in Russia.

The research data emphasizes neurologic, ophthalmologic, endocrine dysfunctions, disorders of genitourinary tract, and other symptoms referring to clinical presentation of neurosarcoidosis [68]. The study of Ruocco \& Lacy (2010) stated that thecognitive functions have declinedin case of neurosarcoidosis [5]; Beste et al. (2015) examined the influence of fatigue on the cognitive functions decline as one of the key neurosarcoidosis manifestations [4]; Mariani \& Shammi (2009) represented the neuropsychological analysis of qualitative-dynamic disorders of mnemonic functions in patients with neurosarcoidosis [3]. Thus, we highlight the central nervous system involvement (more precisely the cerebral cortex) into the pathological process, fragmentary or lack of information describing psychophysiological and neuropsychological manifestations in the clinical presentation of neurosarcoidosis. Specifically, social maladaptation, neurophysiological and neurocognitive symptoms of neurosarcoidosis cause in the first instance maladjustment in the patients.

The purpose of the study is to identify typical and specific features of neurosarcoidosisby analyzing the clinical case.

\section{Case report}

In January 2016, a 26-year-old the patient M was admitted to neurological ward with complaints of recurrent headaches, localized mainly in the temporal-parietal region, numbness and weakness in his left hand, speech disorders.

\section{Anamnesis}

In 2012 patient $\mathrm{M}$ was diagnosed with pulmonary sarcoidosis and intrathoracic lymphatic nodes, in active stage. The patient was treated with prednisolone and omeprazole under the supervision of pulmonologist. At the admission time, the neurological status was characterized by the asymmetry of nasolabial folds, mild hypoesthesia in the left hand. The patient had speech disorders. Arterial hypertension was revealed. According to the magnetic resonance imaging (MRI) of the brain, there were magnetic resonance (MR) images of ischemic cerebrovascular accidents of the right parietal lobe in the right middle cerebral artery (MCA), MR-picture: cystic glial changes in the left parietal lobe, signs ofemerging lacunar postischemic cysts of the right hemisphere of the cerebellum (Figure 1A). The same year, he was treated for acute ischemic stroke in Kursk regional hospital with the diagnosis of transient ischemic attack in the right MCA. To exclude specific brain injury thepatient was transferred to the neurological 
department of the Kursk regional hospital where the diagnosis of neurosarcoidosis with granulomatous lesions of the brain substance with left-sided pyramidal symptoms was redefined. After successful treatment with antioxidants, glucocorticosteroids, neuroprotective and vasoactive drugs patient was discharged with improvement.

\section{Secondary hospitalization in April 2016}

The neurological status of the patient included nasolabial folds, mild hyperesthesia in the left arm, during the hospitalization. The patient complained of sleep disturbance, problems with eating regimeand reduced training activity in comparison with the previous period. The patient underwent MRI (MR spectroscopy) of the brain, rheoencephalography, electrocardiography, and neuropsychological investigation of higher mental functions.

A number of neuropsychological tests are presented in Table 1. Quantitative assessment of the neuropsychological tests performance was carried out according to four parameters: accuracy of performance, pace of performance, coordination of movements during the test performance, movements' differentiation. Each of the parameters was assigned to the corresponding point on the scale proposed by L.I. Vasserman (1997):

- 0 point meant no errors or "non-specific" errors, including appropriate mistakes for healthy patients such as the spelling errors in writing and others.

- 1 point indicated slight disorders, with a number of minor errors corrected by the testee without experimenter's guidance. It was lower limit of anormative line.

-2 points indicated moderate disorders of higher mental functions: the testee was able to perform the task after several attempts by means of detailed prompts and suggestive questions.

-3 points indicated severe disorders of higher mental functions: the task was impossible to perform by the testee even after multiple detailed explanations given by the experimenter.

\section{Results of functional diagnostics}

Electrocardiography examination revealed disorders of the repolarization process. MRI of the brain showed the areas of increased intensity in T2-weighed brain MR images, the hyperintensive and hypointensitve areas in T1-weighed images of the brain in the right hemisphere of the cerebellum and cerebral hemispheres. The obtained data correspond to the positive dynamics of the centers of the right hemisphere of the cerebellum and cortex.

We carried out a series of control T1, T2-weighed images of the sub and supratentorial brain structures in the sagittal and axial projections (Figure 1B)

Areas of increased intensity in T2-weighed images, hypointensity areas in T1-weighed images have been detected in the right hemisphere of the cerebellum and the right parietal lobe. Median structures have not been displaced. The ventricular system had normal shape and size. Subarachnoid spaces of the brain hemispheres were not expanded. Basal cisternal space has not been deformed. Craniovertebral junction was normal, without any peculiarities. The obtained data showed the dynamics of the lesion in the right hemisphere of the cerebellum and the right parietal lobe.

One of the data obtained by methods of functional diagnostics of the patient has been diagnosed with neurosarcoidosis with granulomatous lesions of the brain substance with left-sided pyramidal symptoms-based.

\section{Results of neuropsychological diagnostics}

To study the functional features of the higher mental functions of the patient with neurosarcoidosis neuropsychological diagnostics has been carried out.

The study of the brain lateral organization profile has revealed right-handedness (left hemisphere lateral organization profile).
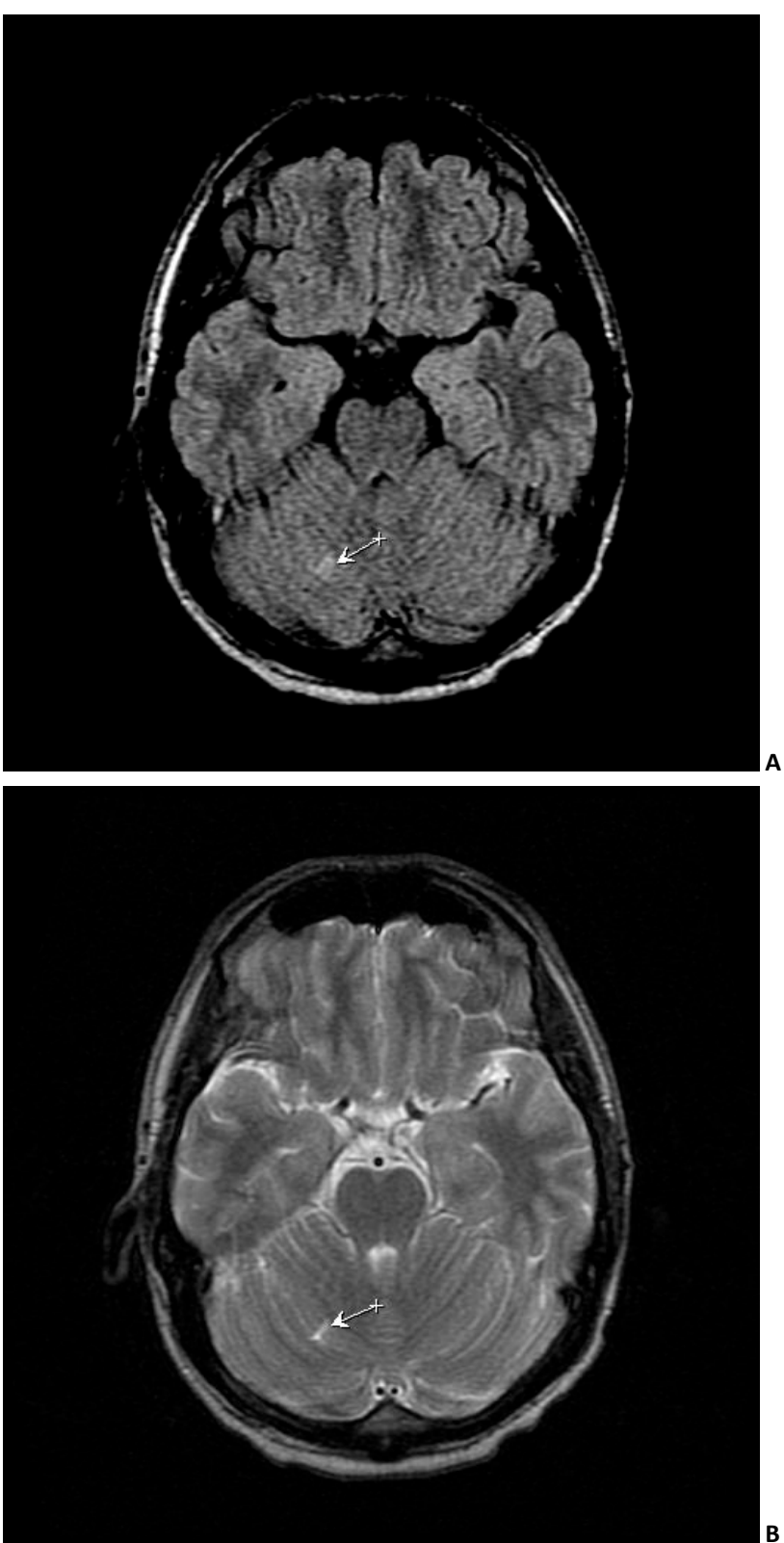

Figure 1. The MRI of the brain (neurosarcoidosis) on primary (A) and secondary (B) hospitalization. 
Table 1. Neuropsychological diagnosis. Functional disorders of higher mental functions in patients with neurosarcoidosis

\begin{tabular}{ll}
\hline \multicolumn{1}{c}{ Name } & The testee is asked: \\
i) to show his left hand; \\
ii) to show the right leg of the experimenter; \\
iii) to show the right hand of the experimenter, sitting with crossed arms;
\end{tabular}

Distinction between the right and left sides according to drawings (for preschool and primary school age). Demonstration of body parts and face (for preschool and primary school age)

iv) to show a desk with the right hand (to the left of a subject there is desk), etc

The experimenter asks a child to draw himself up to his full height without any restrictions. Then a child is asked to show on it, where is the right arm, the left one or leg. If there are errors, a similar request is repeated in respect of own body - a child is asked to show the left and right limbs on himself. If illustration is pictorial or involves some objects or other people, or animals, the experimenter may ask additional questions about their location towards «main character» and to each other.

The experimenter calls various body parts (eyes, elbow, eyebrows, elbows, chin, eyelashes, shoulder, etc.), and requests the testee to show them on himself.

Another variant of this test is one in which the emphasis shifts to assessing location of body parts and faces relative to each other. For example, N.Y. Semago and M.M. Semago offer the following instructions and procedures: "Close your eyes and tell me what you have under/above eyes, under/over chin, over forehead, under lips". "What is to the side of the nose, to the side of ear", "above the shoulders", "...under the neck," “...under the knees", etc. In a situation when the child is difficult to perform the task with his closed eyes, he can do this by relying on the visual analyzer.

Head's Probe (visual form) In the simplest case, sitting in front of the testee, the experimenter, offers him to play backposition one of his hand (the right hand - for the right one, the lefthand - for the left one, without mirror repetition) - frontal, sagittal or horizontal. In a more complex series of the test, the testee has to repeat the position of the hand, touching the same or opposite ear or eye. In sensitized test, the testee has to imitate the position of both hands simultaneously, whilst the right hand touches the nose, and the left one - right ear, etc. Every time the testee is faced with the necessity to overcome the tendency to make mirror poses. There are other variants of the relative position of the hands and body:

i) "voting";

ii) hand horizontally in front of the chest;

iii) hand horizontally under the chin;

iv) the left hand - right cheek;

v) left fist under the right of the hand edge;

vi) the right hand - the left ear. Lefthand - right cheek.

Head's Probe

(verbalform)

\section{Reproduction of}

handposition in relation to

face (D. I. Vassermanetal.)

Transfer pose from one hand to another one

Reproduction of relative position of both wrists

\section{The testee performs the same movements under the verbal instructions.}

Despite the apparent similarity to the previous form, the test is addressed to other mental functions - It evaluates concepts "right - left" and verbal regulation of motor acts.

Sitting in front of the testee, the experimenter shows on himself or demonstrates poses with one of his hands disposed in a certain way toward face according to six pre-prepared pictures. The testee has to repeat them by himself. In fact, it is a concise case of Head's probe.

The researcher gives the testee's hand a particular position and asks to repeat the same gesture with the other hand without opening his eyes.

Standing in front of the testee the experimenter showson himself or demonstrates few positions of hands according to the pre-prepared pictures, the subject has to imitate experimenter's movements.

One part of the tests requires orientation in the spatial position by one hand, and another one - by both hands. In a simple version of this test, the experimenter is near the testee, thereby removing for the latter the need to recode mentally the image from the right hand to the left one and vice versa.

Filling in missing body parts The testee is given several pictures with the schematic (as usual) images of face and body which are lack of some anatomical fragments (nose, lips, eyes, ear, hands, feet, etc.). The testee is asked to fill in the missing body part.

Demonstration of fingers by imitation The testee is asked to repeat a few postures of the hand (stretch II and III, II and V fingers, fold his fingers into a ring, put the second and third fingers at each other, etc.). It is preferable that a person controls movement visually as little as possible, until shielding of the "working hand" from the eyes become possible. Studies are carried out for both hands.
Demonstration of fingersthrough imitation(child version)

A child is asked, without looking at his hand, followed by experimenter to imitate various configurations of fingers.

a) all the fingers straight and closed, palm facing forward;

b) I finger raised upward, the rest gathered in a fist;

c) II thumb straightened, the rest gathered in a fist;

d) II and III fingers arranged in the form of letter $\mathrm{V}$;

e) II and $V$ fingers straightened, the rest gathered in a fist;

f) II and III fingers crossed, the rest gathered in a fist;

g) II and III fingers are straight and arranged in the form of letter V, and I, IV and V in the assembled pinch fingers ("bunny");

h) I and II fingersform a circle,otherfingers are straight.

Demonstration of fingers according verbal instruction (childversion) The testee gets verbal instructions, according to which he must blindly show consistently: the index finger, little finger, middle finger, thumb, little finger, ring finger. Test can be carried out alternately on the right and the left hand and the exemplary scheme: right hand - V I III II IV I IV II; left hand - II IV I V III IV II III I.

A version of this sample: the testees are asked to touch a table with fingers instead of showing the appropriate ones.

Naming the fingers 作 are shown alternately, first time on the right hand, then on the left one, each time changing the position of the hand in space. 
Examination of praxis functions (kinetic, kinesthetic, dynamic, and spatial) has revealed disturbances of pace and accuracy in performance of the movements and actions.

Functional neuropsychological tests were carried out with minor errors (slow performance, inaccurate performance with selfcorrection). When performing movements of a non-dominant (left) hand, the patient made significant errors in accuracy. The patient found it difficult to repeat the movement, he couldn't correct these errors. The reduced movements rate and absence of accuracy was revealed in the process of reproduction (of poses according to the given pattern). In norm, the patient reproduced the necessary poses on a tactile pattern after the pause of 8-10 seconds determining the desired posture by selection method.

The study of dynamic praxis with the use of the "Fence" test has revealed major axis shift in the direction from top to bottom, jitter of the line during the tasks performance with both left and right hands. Micrographyhas been observed while performing the task with the right hand and it indicated the signs of exhaustion.

Reciprocal coordination probe has revealed reduced pace and accuracy confirmed by «blurring» movements and slower fulfillment of the tasks, compared with the same activity according to the given pattern during the test performance. Performing the functional "first-edge-palm" test the patient fulfilled three repeated actions correctly and then he skipped the middle link ("edge") during the fourth and fifth repetitions regardless of the task performance with the right or the left hand (the impairments have been expressed more intensively). It indicated symptomatic of the motor act program and exhaustion as well. The use of the Head probe which is a simple variant of the sensitized variant with double conversion hasn't revealed any impairments of the spatial praxis. The impairments in accuracy and the pace of the tasks fulfillment by the leading and non-dominant hand have not been revealed.

The study of gnostic functions (color gnosis, visual gnosis) conducted with the use of functional test (recognition of the realistic, overlapping and crossed images) have not revealed any impairments of the function. The study of spatial gnosis which was carried out with the use of functional tests supposing recognition of mirror images of the letters has revealed insignificant decrease of the rate and accuracy of the letter recognition. The study of the tactile gnosis has revealed insignificant decrease in the accuracy of touch on the right hand and impossibility of the touch on the left hand. The study of the differential sensitivity on the back of the hand, fingers and forearm with Weber's compass determined touch discrimination of $15 \mathrm{~mm}$ on the left arm. On the hand and forearm of the right hand, it is $10 \mathrm{~mm}$ and on the fingers of the right hand, it is $5 \mathrm{~mm}$. Preservation of innervation on the left hand of up to 1.5 seconds caused by previous touch was revealed.

Impairment of optical spatial representations was detected. While performing functional tests "Blind watch" the patient made mistakes in marking the specified time on the circle, as well as in recognizing time on the pictured dial with arrows and without numbers.

The study of attention stability and concentration using Schulte tables revealed no disturbances (running time is $47 \mathrm{~s}-42 \mathrm{~s}$ $-43 \mathrm{~s}$ ). There are no violations in the study of counting operation. The study of short-term auditory-verbal memory span by using the method "10 words" without interference revealed the decline; remembering curve is as follows: 6-7-7-8 words. In the study by interfering influence, the capacity of short-term auditory-verbal memory was 5 words, indicating reduction of the memory consolidation process. In the study of mental operation stifling errors were identified, emphasizing the decrease in the accuracy of the generalization and comparison processes. There is also the decrease in the level of general knowledge.

The study of the anticipation functions by the "Tower of Hanoi" test showed the increase in the number of used operations in each of three attempts to perform the task: $1^{\text {st }}$ trial -37 operations; $2^{\text {nd }}$ trial -43 operations; $3^{\text {rd }}$ trial -51 operations (normative number of operations is equal to 14). The obtained data highlighted the decrease of anticipation prognostic functions.

The result of neuropsychological assessment revealed impairments of the kinetic and kinesthetic organization of movements (as verified by the reduction in the pace and accuracy of appropriate neuropsychological functional tests); disturbances of the spatial organization of movements and actions, as well as optical spatial gnostic functions. There are disturbances of differential sensitivity. The obtained results may indicate functional disorders of the programming block, regulation and control (prefrontal cortex), as confirmed by disorders of dynamic praxis, as well as by disturbances of anticipative-prognostic functions. Distortions of the optical-spatial representations and spatial organization of movements and actions (praxis) allow us to conclude that there are functional disorders of the posterior parieto-temporo-occipital associative areas of the cerebral cortex with predominate shift to inferior parietal lobe of the right hemisphere.

\section{Discussion}

At present the queries on the clinical cases of sarcoid vasculitis in the Web of Science system have been obtained five. Three out of five cases describe the child's sarcoid vasculitis as a secondary manifestation of the disease [9-11]. The description of the clinical case of a 38-year-old patient with extensive infarction in the brainstem and thalamus, presumably as a result of sarcoid vasculitis, is described as a persistent vegetative state he did not recover from [12]. When the authors conclude that MRI and cerebrospinal fluid (CSF) studies are sensitive to the detection of central nervous system inflammation, but have no specificity, which makes the establishment of neurosarcoidosis a clinical problem. The clinical case does not contain a neurocognitive evaluation of the patient's status. The clinical case of a 41-year-old African American man who was diagnosed with neurosarcoidosis and the symptoms of hypothermia of the center of incontinence, lack of balance and cases of loss of consciousness, contains a medicamentous treatment regime and does not have a description of the neurocognitive status.

Our case's clinical presentation is unique because of the fact that neuropsychological manifestations of neurosarcoidosis have been described at the level of different functional systems (praxis, cognitive, mnestic and regulative functions). On the one hand, the detailed results of the neurological examination (quantitative and qualitative assessment of the manifestations of the disease) are not represented at functional level, because studies explore the neuropsychological manifestations of neurosarcoidosis. On the other hand, studies include the evaluation of either cognitive [4, 5], or memory functions [3] in terms of their reduction, but do not include analysis of other functional systems (praxis, regulatory functions). 


\section{Conclusion}

Thus, based on the received results it has been stated that typical manifestations of neurosarcoidosis are disturbances of motor functions, occurring in the loss of pace and accuracy at performing functional neuropsychological tests aimed to study functions of the kinetic and kinesthetic praxis. The abovementioned neuropsychological disorders at the functional level confirmed the manifestations of peripheral neuropathy and myopathy, which are the clinical manifestations of neurosarcoidosis. Specific manifestations of neurosarcoidosis have various degree of intensity and are determined by localization of granulomas.

In the current clinical case specific manifestations of neurosarcoidosis with predominant localization of granulomas in the cortex of the right parietal lobe and cerebellum demonstrate themselves by the reduction in pace and accuracy of movements and spatial organization of motor functions. At the neurofunctional level of neurosarcoidosis manifestations, there are violations of general motor activity (in the form of peripheral neuropathy and myopathy), the functions of fine motor skills are usually not affected. Atypical manifestations of neurosarcoidosis with small-focal right-handed localization of granulomas are violations of prognostic functions and the reduced consolidation of memory process, related to the morphological base indirectly and possessing high compensatory resource, due to the localization of lesions in the subdominant (right) hemisphere.

Conflict of interest: none declared.

\section{References}

1. Suslina ZA, Kistenev BA, Maksimova MYu, Morgunov VA. Neurosarcoidosis. Moscow, Russia: MEDpress-inform, 2009; 208 p. Russian.

2. Levin OS, Dzampaev AS. Diagnosis and treatment of neurosaroidosis. Almanac of Clinical Medicine 2006; (13): 113-121. Russian. https://elibrary.ru/item.asp?id=9475693.

3. Mariani M, Shammi P. Neurosarcoidosis and associated neuropsychological sequelae: a rare case of isolated intracranial involvement. Clin Neuropsychol 2010; 24(2): 286-304. https://dx.doi.org/10.1080/13854040903347942.

4. Beste C, Kneiphof J, Woitalla D. Effects of fatigue on cognitive control in neurosarcoidosis. Eur Neuropsychopharmacol 2015; 25(4): 522-530. https://dx.doi.org/10.1016/j.euroneuro.2015.01.012.

5. Ruocco AC, Lacy M. Neuropsychological findings in a case of neurosarcoidosis. J Neuropsychiatry Clin Neurosci 2010; 22(1): 123.E36. https://dx.doi.org/10.1176/appi.neuropsych.22.1.123-w.e36.

6. Poddubnyy AF. Clinical and laboratory diagnosis of sarcoidosis. Med gazeta "Zdorov'e Ukrainy» 2000; (11): 8-11. http://sarcoidosis.stormway.ru/rus/sarc kiev.htm.

7. Bullmann C, Faust M, Hoffmann A, Heppner C, Jockenhovel F, MullerWieland D, Krone W. Five cases with central diabetes insipidus and hypogonadism as first presentation of neurosarcoidosis. Eur J Endocrinol 2000; 142: 365-372. https://dx.doi.org/10.1530/eje.0.1420365.

8. Stern R., Akmsamit A., Cifford D, Scott TF; Neurosarcoidosis Study Group. Neurologic presentations of sarcoidosis. Neurol Clin 2010; 28: 185-198. https://doi.org/10.1016/j.ncl.2009.09.012.

9. Scholten V, ten Hove WM, Macdonald EA. An unusual presentation of neurosarcoidosis in an 11-year-old boy. Can J Neurol Sci 2009; 36(6): 783-786. https://www.ncbi.nlm.nih.gov/pubmed/19960762.

10. Anand G, Sin FE, Soilleux E, Chandra J. Isolated paediatric neurosarcoidosis presenting as epilepsiapartialis continua: a case report and review of literature. Eur J Paediatr Neurol 2013; 17(5): 429436. https://dx.doi.org/10.1016/j.ejpn.2013.03.005.

11. Konrad D, Gartenmann M, Martin E, Schoenle EJ. Central diabetes insipidus as the first manifestation of neurosarcoidosis in a 10-year-old girl. Horm Res 2000; 54(2): https://doi.org/10.1159/000053239.

12. Pawate S., Moses H., Sriram S. Presentations and outcomes of neurosarcoidosis: a study of 54 cases. QJM 2009; 102(7): 449-460. https://dx.doi.org/10.1093/qjmed/hcp042.

\section{Authors:}

Vera B. Nikishina - Doctor of Psychology, Head of the Department of Psychology of Health and Correctional Psychology Kursk State Medical University, Kursk, Russia. http://orcid.org/0000-0003-2421-3652.

Yuliya V. Zolotukhina - Candidate of Psychological Sciences, the head of the Department of Psychophysiological Assessment, Kursk Medical Sanitary Unit of the Ministry of Internal Affairs of the Russian Federation, Kursk, Russia. http://orcid.org/0000-0003-3316-0126.

Sergey A. Sirotkin - the head, Kursk Medical Sanitary Unit of the Ministry of Internal Affairs of the Russian Federation, Kursk, Russia. http://orcid.org/0000-0001-8901-9696.

Vitaliy B. Laskov - Doctor of Medical Sciences, Head of the Department of Neurology and Neurosurgery, Kursk State Medical University, Kursk, Russia. http://orcid.org/0000-0003-2870-4547.

Ekaterina A. Petrash - Candidate of Psychological Sciences, Associate Professor of the Department of Health Psychology and Correctional Psychology, Kursk State Medical University, Kursk, Russia. http://orcid.org/0000-0002-3177-088X. 